\title{
Semi-Symmetric Metric Connection on Homothetic Kenmotsu Manifolds
}

\author{
L. Thangmawia, R. Kumar ${ }^{*}$ \\ ${ }^{1}$ Department of Mathematics, Pachhunga University Collage, Aizawl, Mizoram, India
}

Received 12 August 2019, accepted in final revised form 17 November 2019

\begin{abstract}
The object of the paper is to study homothetic Kenmotsu manifold with respect to semisymmetric metric connection. We discuss locally $\varphi$-symmetric homothetic Kenmotsu manifold and $\xi$-projectively flat homothetic Kenmotsu manifold with respect to semisymmetric metric connection. Finally, we construct an example of 3-dimensional homothetic Kenmotsu manifold to verify some results.
\end{abstract}

Keywords: Homothetic Kenmotsu manifold; Semi-Symmetric metric connection; Locally $\varphi$-symmetric manifold; $\xi$-projectively flat.

(c) 2020 JSR Publications. ISSN: 2070-0237 (Print); 2070-0245 (Online). All rights reserved. doi: http://dx.doi.org/10.3329/jsr.v12i3.42601

J. Sci. Res. 12 (3), 223-232 (2020)

\section{Introduction}

A trans-Sasakian manifold of type $(0,0),(\alpha, 0)$ and $(0, \beta)$ are respectively called the cosympletic, $\alpha$-Sasakian and $\beta$-Kenmotsu manifold, $\alpha, \beta$ being scalar functions [1]. In particular if $\alpha=0, \beta=1 ; \alpha=0, \beta$ is non-zero constant and $\alpha=1, \beta=0$ then the trans-Sasakian manifold will be a Kenmotsu manifold; homothetic Kenmotsu manifold and $\alpha$-Sasakian manifold respectively [2]. As $\beta$ is a constant function, $\beta$-Kenmotsu manifolds provide a large variety of Kenmotsu manifolds. $\beta$-Kenmotsu manifold have been studied by several authors such as Shaikh and Hui [3,4], De [5] and many others.

In 1924, Friedman and Schouten [6] introduced the idea of semi-symmetric linear connection on a differentiable manifold. In this continuation, Hayden [7] introduced a semi-symmetric metric connection on a Riemannian manifold. A linear connection $\bar{\nabla}$ in an $n$-dimensional differentiable manifold $M$ is said be a semi-symmetric connection [6] if its torsion tensor $T(X, Y)=\bar{\nabla}_{X} Y-\bar{\nabla}_{Y} X-[X, Y]$ satisfies the following condition

$$
T(X, Y)=\eta(Y) X-\eta(X) Y,
$$

where $\eta$ is a 1 -form associates with vector field $\xi$ as

$$
\eta(X)=g(X, \xi)
$$

for all vector field $X, Y \in \chi(M), \chi(M)$ is the set of all differentiable vector field on $M$.

\footnotetext{
*Corresponding author: rajesh_mzu@yahoo.com
} 
In addition to (1.1), a semi-symmetric connection $\bar{\nabla}$ is called semi-symmetric metric connection [7], if it satisfies

$(\bar{\nabla} g)(Y, Z)=0$,

A relation between the semi-symmetric metric connection $\bar{\nabla}$ and the Levi-Civita connection $\nabla$ on $(M, g)$ is given by [8]

$$
\bar{\nabla}_{X} Y=\nabla_{X} Y+\eta(Y) X-g(X, Y) \xi,
$$

We also have

$$
\left(\bar{\nabla}_{X} \eta\right)(Y)=\left(\nabla_{X} \eta\right) Y-\eta(X) \eta(Y)+\eta(\xi) g(X, Y) .
$$

Let $\bar{R}$ be the curvature tensor of semi-symmetric metric connection $\bar{\nabla}$ given by

$$
\bar{R}(X, Y) Z=\bar{\nabla}_{X} \bar{\nabla}_{Y} Z-\bar{\nabla}_{Y} \bar{\nabla}_{X} Z-\bar{\nabla}_{[X, Y]},
$$

which on using equation (1.4) and (1.5) gives

$$
\bar{R}(X, Y) Z=R(X, Y) Z+\Theta(X, Z) Y-\Theta(Y, Z) X+g(X, Z) Q Y-g(Y . Z) Q X,
$$

where $R(X, Y) Z=\nabla_{X} \nabla_{Y} Z-\nabla_{Y} \nabla_{X} Z-\nabla_{[X, Y]} Z$,

is the Riemannian curvature tensor of connection $\nabla, \Theta$ is a tensor field of type $(0,2)$ and $Q$ is a tensor field of type $(1,1)$ is given by

$$
\Theta(Y, Z)=g(Q Y, Z)=\left(\nabla_{Y} \eta\right) Z-\eta(Y) \eta(Z)+\frac{1}{2} \eta(\xi) g(Y, Z) .
$$

From (1.7) and (1.8), we obtain

$$
\begin{aligned}
& \quad \bar{R}(X, Y, Z, W)={ }^{\prime} R(X, Y, Z, W)-\Theta(Y, Z) g(X, W)+\Theta(X, Z) g(Y, W) \\
& -\Theta(X, W) g(Y, Z)+\Theta(Y, W) g(X, Z),
\end{aligned}
$$

Where ' $\bar{R}(X, Y, Z, W)=g(\bar{R}(X, Y) Z, W)$ and ' $R(X, Y, Z, W)=g(R(X, Y) Z, W)$.

The semi-symmetric metric connection in a Kenmotsu manifold was studied by Tripathi [9], Bagewadi et al. [10], De and Pathak [11]. In a recent paper Barman [12] studied the semi-symmetric metric connection in Lorentzian $\alpha$-Sasakian manifold. The calculation used to find the results of semi-symmetric metric connection was also used by Eshghi [13] and Moniruzzaman et al. [14] in tensor notation form.

\section{Homothetic Kenmotsu Manifolds}

A $(2 n+1)$-dimensonal differentiable manifold $M^{2 n+1}$ is said to be an almost contact metric manifold [15] if it admits a $(1,1)$ tensor field $\varphi$, a vector field $\xi$, a 1-form $\eta$ and a Riemannian metric $g$ which satisfies

$$
\begin{aligned}
& \qquad \varphi \xi=0, \eta(\varphi X)=0, \eta(\xi)=1, g(X, \xi)=\eta(X), \\
& \varphi^{2} X=-X+\eta(X) \xi \\
& \quad g(\varphi X, \varphi Y)=g(X, Y)-\eta(X) \eta(Y), \\
& \text { for all vector field } X, Y \text { on } M^{2 n+1} \text {. }
\end{aligned}
$$


An almost contact metric manifold $M^{2 n+1}(\varphi, \xi, \eta, g)$ is said to be $\beta$-Kenmotsu manifold if the following conditions hold:

$$
\left(\nabla_{X} \xi\right)=\beta[X-\eta(X) \xi]
$$

and $\left(\nabla_{X} \varphi\right) Y=\beta[g(\varphi X, Y) \xi-\eta(X) \varphi(Y)]$,

where $\nabla$ denotes the Riemannian connection of $g$. If $\beta=1$ then $\beta$-Kenmotsu manifold is called Kenmotsu manifold and if $\beta$ is constant then it is called homothetic Kenmotsu manifold.

In a homothetic Kenmotsu manifold the following relations hold [2]

$$
\begin{aligned}
& \left(\nabla_{X} \eta\right) Y=\beta[g(X, Y)-\eta(X) \eta(Y)], \\
& \eta(R(X, Y) Z)=\beta^{2}[g(X, Z) \eta(Y)-g(Y, Z) \eta(X)], \\
& R(\xi, X) Y=\beta^{2}[\eta(Y) X-g(X, Y) \xi] \\
& R(\xi, X) \xi=\beta^{2}[X-\eta(X) \xi] \\
& R(X, Y) \xi=\beta^{2}[\eta(X) Y-\eta(Y) X] \\
& S(X, \xi)=-2 n \beta^{2} \eta(X), \\
& Q \xi=-2 n \beta^{2} \xi
\end{aligned}
$$

where $S$ is the Ricci tensor of the Levi-Civita connection and $Q$ is the Ricci operator.

\section{Curvature Tensor of A Homothetic Kenmotsu Manifold with respect to the Semi Symmetric Metric Connection}

Using (2.1) and (2.6) in (1.8), we get

$$
\Theta(Y, Z)=g(Q Y, Z)=\beta g(X, Y)-(\beta+1) \eta(X) \eta(Y)+\frac{1}{2} g(X, Y),
$$

It follows, that

$$
Q(X)=\left(\beta+\frac{1}{2}\right) X-(\beta+1) \eta(X) \xi
$$

By virtue of (3.1) and (3.2), equation (1.7) reduce to the form

$$
\begin{aligned}
& \bar{R}(X, Y) Z=R(X, Y) Z+(2 \beta+1)[g(X, Z) Y-g(Y, Z) X] \\
& -(\beta+1)[g(X, Z) \eta(Y) \xi-g(Y, Z) \eta(X) \xi+\eta(X) \eta(Z) Y-\eta(Y) \eta(Z) X],
\end{aligned}
$$

which is the relation between curvature tensors connection $\bar{\nabla}$ and $\nabla$.

Taking the inner product of (3.3) with $W$, we get

$$
\begin{aligned}
& ' \bar{R}(X, Y, Z, W)=^{\prime} R(X, Y, Z, W)+(2 \beta+1)[g(X, Z) g(Y, W)-g(Y, Z) g(X, W)] \\
& -(\beta+1)[g(X, Z) \eta(Y) \eta(W)-g(Y, Z) \eta(X) \eta(W)+g(Y, W) \eta(X) \eta(Z)- \\
& g(X, W) \eta Y \eta(Z),
\end{aligned}
$$

Putting $X=Y=e_{i}$ in the above equation and taking summation over $i, 1 \leq i \leq n$, we get

$$
\bar{S}(Y, Z)=S(Y, Z)-(4 n-1) \beta g(Y, Z)-(2 n-1) g(Y, Z)+(2 n-1)(\beta+
$$

1) $\eta(Y) \eta(Z)$

where $\bar{S}$ and $S$ are the Ricci tensors of the connection $\bar{\nabla}$ and $\nabla$ respectively. Putting $Z=\xi$ in (3.5) and using (2.1) and (2.11), we get

$$
\bar{S}(Y, \xi)=-2 n \beta(\beta+1) \eta(Y) .
$$


Thus we can state the following theorem:

Theorem 3.1. In a homothetic Kenmotsu manifold $M^{2 n+1}$ with respect to the semisymmetric metric connection $\bar{\nabla}$

(i): the curvature tensor $\bar{R}$ is given by

$$
\begin{aligned}
& ' \bar{R}(X, Y, Z, W)={ }^{\prime} R(X, Y, Z, W)+(2 \beta+1)[g(X, Z) g(Y, W)-g(Y, Z) g(X, W)] \\
& -(\beta+1)[g(X, Z) \eta(Y) \eta(W)-g(Y, Z) \eta(X) \eta(W)+g(Y, W) \eta(X) \eta(Z)- \\
& g X, W \eta Y \eta(Z),
\end{aligned}
$$

(ii): the Ricci tensor $\bar{S}$ is given by

$$
\begin{aligned}
& \bar{S}(Y, Z)=S(Y, Z)-(4 n-1) \beta g(Y, Z)-(2 n-1) g(Y, Z)+(2 n-1)(\beta+ \\
& 1 \eta Y \eta(Z),
\end{aligned}
$$

(iii): the Ricci tensor $\bar{S}$ is symmetric,

(iv): $\bar{S}(Y, \xi)=-2 n \beta(\beta+1) \eta(Y)$.

Taking the cyclic permutation of $X, Y$ and $Z$ in (3.3), we get

$$
\begin{aligned}
& \bar{R}(Y, Z) X=R(Y, Z) X+(2 \beta+1)[g(Y, X) Z-g(Z, X) Y] \\
& -(\beta+1)[g(Y, X) \eta(Z) \xi-g(Z, X) \eta(Y) \xi+\eta(Y) \eta(X) Z-\eta(Z) \eta(X) Y],
\end{aligned}
$$

and $\bar{R}(Z, X) Y=R(Z, X) Y+(2 \beta+1)[g(Z, Y) X-g(X, Y) Z]$

$$
-(\beta+1)[g(Z, Y) \eta(X) \xi-g(X, Y) \eta(Z) \xi+\eta(Z) \eta(Y) X-\eta(X) \eta(Y) Z]
$$

Using Bianchi's first identity in the addition of (3.3), (3.7) and (3.8), we get

$$
\bar{R}(X, Y) Z+\bar{R}(Y, Z) X+\bar{R}(Z, X) Y=0 .
$$

Thus we can state the following theorem:

Theorem 3.2. The curvature tensor of a homothetic Kenmotsu manifold $M^{2 n+1}$ with respect to the semi-symmetric metric connection satisfies the equation

$$
\bar{R}(X, Y) Z+\bar{R}(Y, Z) X+\bar{R}(Z, X) Y=0 .
$$

Now, interchanging $X$ and $Y$ in the equation (3.4), we get

$$
\begin{aligned}
& \bar{R}(Y, X, Z, W)=' R(Y, X, Z, W)+(2 \beta+1)[g(Y, Z) g(X, W)-g(X, Z) g(Y, W)] \\
& -(\beta+1)[g(Y, Z) \eta(X) \eta(W)-g(X, Z) \eta(Y) \eta(W)+g(X, W) \eta(Y) \eta(Z)- \\
& g Y, W \eta X \eta(Z) .
\end{aligned}
$$

Using the fact $' R(X, Y, Z, W)+{ }^{\prime} R(Y, X, Z, W)=0$, in the addition of (3.4) and (3.10), we get

$$
' \bar{R}(X, Y, Z, W)+{ }^{\prime} \bar{R}(Y, X, Z, W)=0 .
$$

Again interchanging $Z$ and $U$ in (3.4), we get

$$
\begin{gathered}
\bar{R}(X, Y, W, Z)=R(X, Y, W, Z)+(2 \beta+1)[g(X, W) g(Y, Z)-g(Y, W) g(X, Z)] \\
-(\beta-1)[g(X, W) \eta(Y) \eta(Z)-g(Y, W) \eta(X) \eta(Z)+g(Y, Z) \eta(X) \eta(W)-
\end{gathered}
$$
$g X, Z \eta Y \eta(W)$.

Using the fact ' $R(X, Y, Z, W)+{ }^{\prime} R(X, Y, W, Z)=0$ in the addition of (3.4) and (3.12), we get

$$
' \bar{R}(X, Y, Z, W)+{ }^{\prime} \bar{R}(Y, X, Z, W)=0 .
$$


Again interchanging pair of slots in (3.4), we get

$$
\begin{aligned}
& ' \bar{R}(Z, W, X, Y)={ }^{\prime} R(Z, W, X, Y)+(2 \beta+1)[g(Z, X) g(W, Y)-g(W, X) g(Z, Y)] \\
& -(\beta+1)[g(Z, X) \eta(W) \eta(Y)-g(W, X) \eta(Z) \eta(Y)+g(W, Y) \eta(Z) \eta(X)- \\
& g Z, Y \eta W \eta(X) .
\end{aligned}
$$

Using the fact ${ }^{\prime} R(X, Y, Z, W)-{ }^{\prime} R(Z, W, X, Y)=0$ in the subtraction of (3.14) from (3.4), we get

$$
' \bar{R}(X, Y, Z, W)-' \bar{R}(Z, W, X, Y)=0 .
$$

Thus in the view of (3.11), (3.13) and (3.15), we can state the following:

Theorem 3.3. The curvature tensor of type $(0,4)$ of a semi-symmetric metric connection in a homothetic Kenmotsu manifold is

(i): skew-symmetric in first two slots,

(ii): skew-symmetric in last two slots,

(iii): symmetric in pair of slots.

Next, Let us consider $\bar{R}(X, Y) Z=0$, then by virtue of (3.3), we have

$$
\begin{aligned}
& R(X, Y) Z=(2 \beta+1)[g(Y, Z) X-g(X, Z) Y] \\
& +(\beta+1)[g(X, Z) \eta(Y) \xi-g(Y, Z) \eta(X) \xi+\eta(X) \eta(Z) Y-\eta(Y) \eta(Z) X] .
\end{aligned}
$$

Taking the inner product of the above with $\xi$, we get

$$
\eta(R(X, Y) Z)=\beta[g(Y, Z) \eta(X)-g(X, Z) \eta(Y)],
$$

which by virtue of (2.1), we have

$$
R(X, Y) Z=\beta[g(Y, Z) X-g(X, Z) Y] .
$$

Thus the manifold is of constant curvature $\beta$. Hence we can state the following:

Theorem 3.4. If the curvature tensor of a semi-symmetric metric connection in a homothetic Kenmotsu manifold $M^{2 n+1}$ vanishes, then the manifold is of constant curvature $\beta$.

Now, consider the Ricci tensor of a semi-symmetric metric connection $M^{2 n+1}$ vanishes, i.e. $\bar{S}(Y, Z)=0$, from (3.5), we have

$$
S(Y, Z)=2(3 n-1) g(Y, Z)-(2 n-1)(\beta+1) \eta(Y) \eta(Z) .
$$

Thus from above, we can state the following:

Theorem 3.5. If the Ricci tensor of a semi-symmetric metric connection $\bar{\nabla}$ in a homothetic Kenmotsu manifold vanishes, then the manifold $M^{2 n+1}$ is $\eta$-Einstein manifold.

\section{Locally $\varphi$-Symmetric Homothetic Kenmotsu Manifolds with respect to the Semi- Symmetric Metric Connection}

Definition 4.1. A homothetic Kenmotsu manifold $M^{2 n+1}(\varphi, \xi, \eta, g)$ with respect to semisymmetric metric connection is said to be locally $\varphi$-symmetric if and only if

$$
\varphi^{2}\left(\left(\bar{\nabla}_{W} \bar{R}\right)(X, Y) Z\right)=0,
$$


hold for all vector fields $X, Y, Z, W$ orthogonal to $\xi$, that is for any horizontal vector field $X, Y, Z, W$.

This notion was introduced by Takahashi [16] for a Sasakian manifold.

Taking covariant differentiation of (3.3) with respect to $W$, we get

$$
\left(\bar{\nabla}_{W} \bar{R}\right)(X, Y) Z=\bar{\nabla}_{W} \bar{R}(X, Y) Z-\bar{R}\left(\bar{\nabla}_{W} X, Y\right) Z-\bar{R}\left(X, \bar{\nabla}_{W} Y\right) Z-\bar{R}(X, Y) \bar{\nabla}_{W} Z .
$$

Using (1.3), (1.4), (2.1), (2.4), (2.5) and (2.6) in (4.2), we obtain

$$
\begin{aligned}
& \left(\bar{\nabla}_{W} \bar{R}\right)(X, Y) Z=\left(\nabla_{W} R\right)(X, Y) Z-\eta(X) R(W, Y) Z-\eta(Y) R(X, W) Z-\eta(Z) R(X, Y) W \\
& \quad-R(X, Y, Z, W) \xi+\left(\beta^{2}-2 \beta-1\right)[g(X, Z) g(Y, W) \xi-g(Y, Z) g(X, W) \xi \\
& \quad+g(X, W) \eta(Z) Y-g(W, Y) \eta(Z) X+g(W, Z) \eta(X) Y-g(W, Z) \eta(Y) X] \\
& +(\beta+1)^{2}[g(X, Z) \eta(Y)-g(Y, Z) \eta(X)] \eta(W) \xi+\beta[g(Y, Z) \eta(X) W- \\
& \quad g X, Z \eta Y W \\
& \quad+2(\beta+1)[\eta(X) Y-\eta(Y) X] \eta(Z) \eta(W) .
\end{aligned}
$$

Applying $\varphi^{2}$ on both the side of (4.3) and using (2.1) and (2.2), we have

$$
\begin{aligned}
& \quad \varphi^{2}\left(\left(\bar{\nabla}_{W} \bar{R}\right)(X, Y) Z\right)=\varphi^{2}\left(\left(\nabla_{W} R\right)(X, Y) Z\right)+\eta(X) R(W, Y) Z-\eta(X) \eta(R(W, Y) Z) \\
& +\eta(Y) R(X, W) Z-\eta(Y) \eta(R(X, W) Z)+\left(\beta^{2}-\beta-1\right)[-g(X, W) \eta(Z) Y \\
& \quad+g(X, W) \eta(Z) \eta(Y) \xi+g(W, Y) \eta(Z) X-g(W, Y) \eta(Z) \eta(X) \xi-g(W, Z) \eta(X) Y \\
& \quad+g(W, Z) \eta(X) \eta(Y) \xi+g(W, Z) \eta(Y) X-g(W, Z) \eta(Y) \eta(X) \xi]+ \\
& \beta[-g(Y, Z) \eta(X) W+g(Y, Z) \eta(X) \eta(W) \xi+g(X, Z) \eta(Y) W-g(X, Z) \eta(Y) \eta(W) \xi] \\
& \quad+2(\beta+1)[-\eta(X) Y+\eta(Y) X] \eta(Z) \eta(W) .
\end{aligned}
$$

Now, taking $X, Y, Z, W$ orthogonal to $\xi$ in (4.4), we have

$$
\varphi^{2}\left(\left(\bar{\nabla}_{W} \bar{R}\right)(X, Y) Z\right)=\varphi^{2}\left(\left(\nabla_{W} R\right)(X, Y) Z\right) .
$$

Thus we can state the following:

Theorem 4.1. A $(2 n+1)$-dimensional homothetic Kenmotsu manifold is locally $\varphi$ symmetric with respect to the semi-symmetric metric connection if and only if the manifold is also locally $\varphi$-symmetric with respect to the Levi-Civita connection.

\section{5. -Projectively Flat Homothetic Kenmotsu Manifolds with respect to the Semi- Symmetric Metric Connection}

Definition 5.1. The pseudo projective curvature tensor in an almost metric manifold $M^{2 n+1}$ with respect to Levi-Civita connection is defined as follows [17]

$$
P(X, Y) Z=R(X, Y) Z-\frac{1}{2 n}[S(Y, Z) X-S(X, Z) Y] .
$$

The projective curvature tensor is an important tensor in differential geometry. In a Riemannian manifold $M^{2 n+1}$, if there exist a one-to-one correspondence between each coordinate neighborhood of $M^{2 n+1}$ and a domain in Euclidean space such that any geodesic of the Riemannian manifold corresponds to a straight line in the Euclidean space then $M^{2 n+1}$ is said to be locally projectively flat. For recent developments on projective curvature tensor, we refer $[18,19]$. 
Definition 5.2. A homothetic Kenmotsu manifold $M^{2 n+1}$ with respect to the semisymmetric metric connection is said to be $\xi$-projectively flat if

$$
\bar{P}(X, Y) \xi=0,
$$

for all vector fields $X, Y$ orthogonal to $\xi$, where $\bar{P}$ is a projective curvature tensor with respect to semi-symmetric metric connection given by

$$
\bar{P}(X, Y) Z=\bar{R}(X, Y) Z-\frac{1}{2 n}[\bar{S}(Y, Z) X-\bar{S}(X, Z) Y],
$$

where $\bar{R}$ and $\bar{S}$ are the Riemannian curvature tensor and Ricci tensor with respect to the semi-symmetric metric connection $\bar{\nabla}$ respectively. This notion was first defined by Tripathi and Dwivedi [20]. A manifold $M^{2 n+1}$ is called horizontal $\xi$-projectively flat manifold if (5.2) holds for $X, Y$ orthogonal to $\xi$.

Using (3.3) in (5.3), we get

$$
\begin{gathered}
\bar{P}(X, Y) Z=R(X, Y) Z+(2 \beta+1)[g(X, Z) Y-g(Y, Z) X]+(\beta+1)[g(Y, Z) \eta(X) \xi \\
-g(X, Z) \eta(Y) \xi+\eta(Y) \eta(Z) X-\eta(X) \eta(Z) Y]-\frac{1}{2 n}[\bar{S}(Y, Z) X-\bar{S}(X, Z) Y] .
\end{gathered}
$$

Putting $Z=\xi$ and using (2.1), (2.10) and (3.6) in (5.4), we get

$$
\bar{P}(X, Y) \xi=\beta[\eta(X) Y-\eta(Y) X] .
$$

If we consider $X, Y$ orthogonal to $\xi$, then (5.5) reduces to

$$
\bar{P}(X, Y) \xi=0,
$$

we called such a manifold a horizontal $\xi$-projectively flat manifold. Hence we state the following:

Theorem 5.3. A $(2 n+1)$-dimensional homothetic Kenmotsu manifold is horizontal $\xi$ projectively flat with respect to the semi-symmetric metric connection.

Again using (3.5) in (5.4), we get

$$
\begin{aligned}
& \bar{P}(X, Y) Z=P(X, Y) Z+\frac{\beta-1}{2 n}[g(X, Z) Y-g(Y, Z) X]+(\beta+1)[g(Y, Z) \eta(X) \xi \\
& -g(X, Z) \eta(Y) \xi+\eta(Y) \eta(Z) X-\eta(X) \eta(Z) Y] .
\end{aligned}
$$

Putting $Z=\xi$ in (5.6) and using (2.1), we obtain

$$
\bar{P}(X, Y) \xi=P(X, Y) \xi+\frac{(2 n-1)(\beta+1)}{2 n}[\eta(Y) X-\eta(X) Y] .
$$

On taking $X, Y$ orthogonal to $\xi$ in (5.7), we get

$$
\bar{P}(X, Y) \xi=P(X, Y) \xi .
$$

Hence we state the following theorem:

Theorem 5.4. A $(2 n+1)$-dimensional homothetic Kenmotsu manifold is horizontal $\xi$ projective flat with respect to the semi-symmetric metric connection if and only if the manifold is $\xi$-projectively flat with respect to the Levi-Civita connection. 


\section{Example}

In this section we consider coordinate space $R^{3}$ (with coordinate $x, y, z$ ) and calculate the components of curvature tensor with respect to semi-symmetric metric connection, then we verified the theorem (3.2) and (3.5)

Let us consider the 3-dimensional manifold $M=\left\{(x, y, z) \in R^{3}\right\}$ where $(x, y, z)$ are the standard coordinate of $R^{3}$. Let $\left(e_{1}, e_{2}, e_{3}\right)$ are linearly independent vector fields at each point of $M$ and given by

$$
e_{1}=\beta z \frac{\partial}{\partial x}, \quad e_{2}=\beta z \frac{\partial}{\partial y}, \quad e_{3}=\beta z \frac{\partial}{\partial z} .
$$

where $\beta$ is constant. Let $g$ be the Riemannian metric defined by

$$
g=\frac{d x^{2}+d y^{2}+d z^{2}}{\beta^{2} z^{2}}
$$

then we have

$$
\begin{aligned}
& g\left(e_{1}, e_{1}\right)=g\left(e_{2}, e_{2}\right)=g\left(e_{3}, e_{3}\right)=1, \\
& g\left(e_{1}, e_{2}\right)=g\left(e_{1}, e_{3}\right)=g\left(e_{2}, e_{3}\right)=0 .
\end{aligned}
$$

Let $\eta$ be a 1 -form defined by $\eta(U)=g\left(U, e_{3}\right)$ for any vector field $U \in \chi(M)$ and $\varphi$ be the 1-1 tensor field defined as

$$
\varphi e_{1}=e_{2}, \quad \varphi e_{2}=-e_{1}, \quad \text { and } \quad \varphi e_{3}=0 .
$$

Then using the linearity of $\varphi$ on $g$, we have

$\eta\left(e_{3}\right)=1, \varphi^{2} U=-U+\eta(U) e_{3}$ and $g(\varphi U, \varphi W)=g(U, W)-\eta(U) \eta(W)$,

for any vector field $U, W \in \chi(M)$. Thus for $e_{3}=\xi$, the structure $(\varphi, \xi, \eta, g)$ defines an almost contact metric structure on $M$.

Let $\nabla$ be the Riemannian connection of the metric tensor $g$, then we have

$$
\left[e_{1}, e_{2}\right]=0, \quad\left[e_{1}, e_{3}\right]=-\beta e_{1} \quad \text { and } \quad\left[e_{2}, e_{3}\right]=-\beta e_{2} \text {. }
$$

Koszul formula for the Riemannian $\nabla$ of the metric $g$ is given by

$$
\begin{aligned}
& 2 g\left(\nabla_{X} Y, Z\right)=X(g(Y, Z))+Y(g(Z, X))-Z(g(X, Y))-g(X,[Y, Z]) \\
& -g(Y,[X, Z])+g(Z,[X, Y]) .
\end{aligned}
$$

Using above Koszul formula for the Riemannian metric $g$, we can easily calculate

$$
\begin{array}{lll}
\nabla_{e_{1}} e_{1}=\beta e_{3}, & \nabla_{e_{1}} e_{2}=0, & \nabla_{e_{1}} e_{3}=-\beta e_{1}, \\
\nabla_{e_{2}} e_{1}=0, & \nabla_{e_{2}} e_{2}=\beta e_{3}, & \nabla_{e_{2}} e_{3}=-\beta e_{2}, \\
\nabla_{e_{3}} e_{1}=0, & \nabla_{e_{3}} e_{2}=0, & \nabla_{e_{3}} e_{3}=0 .
\end{array}
$$

From above it can be easily seen that the manifold $M(\varphi, \xi, \eta, g)$ satisfies the condition

$$
\left(\nabla_{X} \varphi\right) Y=\beta[g(\varphi X, Y) \xi-\eta(Y) \varphi X], \quad \nabla_{X} \xi=\beta[X-\eta(X) \xi],
$$

where $\beta$ is constant. Hence the structure $(\varphi, \xi, \eta, g)$ is homothetic Kenmotsu structure and the manifold $M$ equipped with homothetic Kenmotsu structure is a homothetic Kenmotsu manifold.

By the use of (6.6) in (1.4), we obtain

$$
\bar{\nabla}_{e_{1}} e_{1}=(\beta-1) e_{3}, \quad \bar{\nabla}_{e_{1}} e_{2}=0, \quad \bar{\nabla}_{e_{1}} e_{3}=-(\beta-1) e_{1},
$$




$$
\begin{array}{ccc}
\bar{\nabla}_{e_{2}} e_{1}=0, & \bar{\nabla}_{e_{2}} e_{2}=(\beta-1) e_{3}, & \bar{\nabla}_{e_{2}} e_{3}=-(\beta-1) e_{2}, \\
\bar{\nabla}_{e_{3}} e_{1}=0, & \bar{\nabla}_{e_{3}} e_{2}=0, & \bar{\nabla}_{e_{3}} e_{3}=0 .
\end{array}
$$

Therefore, the manifold is a homothetic Kenmotsu manifold with respect to the semisymmetric metric connection.

By using the equation (6.6), we can obtain the component of the curvature tensor with respect to Levi-Civita connection as follows:

$$
\begin{array}{ll}
R\left(e_{1}, e_{2}\right) e_{2}=-\beta^{2} e_{1}, & R\left(e_{1}, e_{3}\right) e_{3}=-\beta^{2} e_{1}, \quad R\left(e_{2}, e_{1}\right) e_{1}=-\beta^{2} e_{2}, \\
R\left(e_{2}, e_{3}\right) e_{3}=-\beta^{2} e_{2}, & R\left(e_{3}, e_{1}\right) e_{1}=-\beta^{2} e_{3}, \quad R\left(e_{3}, e_{2}\right) e_{2}=-\beta^{2} e_{3}, \\
R\left(e_{1}, e_{2}\right) e_{3}=0, & R\left(e_{2}, e_{3}\right) e_{2}=-\beta^{2} e_{3}, \quad R\left(e_{1}, e_{2}\right) e_{1}=-\beta^{2} e_{2},
\end{array}
$$

Also the curvature tensor with respect to semi-symmetric metric connection can be obtained by using (6.7) as follows:

$$
\begin{aligned}
& \bar{R}\left(e_{1}, e_{2}\right) e_{2}=-(\beta-1)^{2} e_{1}, \bar{R}\left(e_{1}, e_{3}\right) e_{3}=-\beta(\beta-1) e_{1}, \bar{R}\left(e_{2}, e_{1}\right) e_{1}= \\
& -(\beta-1)^{2} e_{2}, \\
& \bar{R}\left(e_{2}, e_{3}\right) e_{3}=-\beta(\beta-1) e_{2}, \quad \bar{R}\left(e_{3}, e_{1}\right) e_{1}=-\beta(\beta-1) e_{3}, \quad \bar{R}\left(e_{3}, e_{2}\right) e_{2}= \\
& -\beta(\beta-1) e_{3}, \\
& \bar{R}\left(e_{1}, e_{2}\right) e_{3}=0, \quad \bar{R}\left(e_{2}, e_{3}\right) e_{2}=\beta(\beta-1) e_{3}, \quad \bar{R}\left(e_{1}, e_{2}\right) e_{1}=(\beta-1)^{2} e_{2},
\end{aligned}
$$

From the above results of the curvature tensor it follows that

$$
\varphi^{2}\left(\left(\bar{\nabla}_{W} \bar{R}\right)(X, Y) Z\right)=\varphi^{2}\left(\left(\nabla_{W} R\right)(X, Y) Z\right)=0 .
$$

Therefore, this example supports the theorem (4.1).

Using the expression of the curvature tensors with respect to the semi-symmetric metric connection we can obtain the Ricci tensors as follows:

$$
\begin{aligned}
& \bar{S}\left(e_{1}, e_{1}\right)=\bar{S}\left(e_{2}, e_{2}\right)=-(\beta-1)(2 \beta-1), \quad \bar{S}\left(e_{3}, e_{3}\right)=-2 \beta(\beta-1), \\
& \bar{S}\left(e_{1}, e_{2}\right)=\bar{S}\left(e_{1}, e_{3}\right)=\bar{S}\left(e_{2}, e_{3}\right)=0 .
\end{aligned}
$$

In a 3-dimensional homothetic Kenmotsu manifold $M^{3}$, the Projective curvature tensor with respect to the semi-symmetric metric connection is given by

$$
\bar{P}(X, Y) Z=\bar{R}(X, Y) Z-\frac{1}{2}[\bar{S}(Y, Z) X-\bar{S}(X, Z) Y] .
$$

Putting $Z=e_{3}$ in above equation, we get

$$
\bar{P}(X, Y) e_{3}=\bar{R}(X, Y) e_{3}-\frac{1}{2}\left[\bar{S}\left(Y, e_{3}\right) X-\bar{S}\left(X, e_{3}\right) Y\right] .
$$

Let $X$ and $Y$ are any two vector fields given by

$$
X=a_{1} e_{1}+a_{2} e_{2}+a_{3} e_{3} \text { and } Y=b_{1} e_{1}+b_{2} e_{2}+b_{3} e_{3} .
$$

Using (6.11) and above relations, we get

$$
\bar{P}(X, Y) e_{3}=0 \text {. }
$$

Therefore, the manifold is $\xi$-projectively flat on a homothetic Kenmotsu manifold with respect to the semi-symmetric metric connection which verifies the Theorem 5.1. 


\section{Conclusion}

It has been seen that at each point of a differentiable manifold $M$ there is an $n$-dimentional tangent space $T_{p}(M)$ and any two tangent space $T_{p}(M)$ and $T_{q}(M)$ at point $p$ and $q$ respectively are isomorphic. However, in order to obtain a definite isomorphism relating $T_{p}(M)$ and $T_{q}(M)$ it is necessary to introduce some additional structure on the manifold called a connection which connect the different points on the manifold. In this paper we find the curvature tensor, Ricci tensor, scalar curvature tensor and other properties of homothetic Kenmotsu manifold with respect to semi-symmetric metric connection. The result of this paper will be helpful for researchers to develop the new connection on homothetic Kenmotsu manifold. The given example of homothetic Kenmotsu manifold of this paper will be used in the study of Relativity and Quantum mechanics.

\section{Acknowledgment}

The authors are very thankful to the referee for his suggestion in the improvement of the paper.

\section{References}

1. D. E. Blair, Lectures Notes in Mathematics (Springer-Verlag, Berlin, 509, 1976).

2. K. Kenmotsu, Tôhoku Math. J. 24, 2 (1972). https://doi.org/10.2748/tmj/1178241594

3. A. Shaikh and S. K. Hui, Extracta Mathematical 24, 3 (2009). https://doi.org/10.4134/CKMS.2009.24.3.415

4. A. A. Shaikh and S. K. Hui, Publication de Ĺinstitut Mathématique 89, 103 (2011). http://doi.org/10.2298/pim1103077a

5. K. De, On a Class of $\beta$-Kenmotsu Manifolds 29, 2 (2014).

6. A. Friedmann and J. A. Schouten, Math. Zeitschrift 21, 211 (1924). https://doi.org/10.1007/bf01187468

7. H. A. Hayden, Proc. London Math. Soc. 34 (1932). https://doi.org/10.4134/CKMS.2009.24.3.415

8. K. Yano, Revue Roumaine de Math. Pure et Appliques 15, 1570 (1970).

9. M. M. Tripathi, J. Pure Math. 16, 67 (1999).

10. C. S. Bagewadi, D. G. Prakasha, and Venkatesha, Stud. Cercet. Stiint. Ser. Mat. Univ. Bacau. 17, 21 (2007).

11. U. C. De and G. Pathak, Bull. Cal. Math. Soc. 94, 4 (2002).

12. A. Barman, Novi Sad J. Math. 44, 2 (2014).

13. M. Eshghi, J. Sci. Res. 3, 3 (2011). https://doi.org/10.3329/jsr.v3i3.8071

14. M. Moniruzzaman and S. B. Faruque, J. Sci. Res. 11, 2 (2019). https://doi.org/10.3329/jsr.v10i2.32829

15. T. Q. Binh, Period. Math. Hungary 21, 2 (1990). https://doi.org/10.1007/bf01946849

16. T. Takahashi, Tohoku Math. J. 29, 91(1977). https://doi.org/10.2748/tmj/1178240699

17. R. S. Mishra. Chandrama Prakashan (Balrampur House, Allahabad, India, 1984).

18. M. S. Stanković, M. Lj. Zlatanović and N. O. Vesić, Filomat 29, 573 (2015). https://doi.org/10.2298/FIL1503573S

19. Venkatesha and C. S. Bagewadi, Soochow J. Math. 32, 1 (2006).

20. M. M. Tripathi and M. K. Dwivedi, Proc. Indian Acad. Sci. Math. Sci. 118, 371 (2008). https://doi.org/10.1007/s12044-008-0029-1 